\title{
ADAMTS8 targets ERK to suppress cell proliferation, invasion, and metastasis of hepatocellular carcinoma
}

This article was published in the following Dove Press journal:

OncoTargets and Therapy

\author{
Xuetao Zhao' \\ Congrong Yang ${ }^{2}$ \\ Jianhua $\mathrm{Wu}^{3}$ \\ Yuemin $\mathrm{Nan}^{4}$ \\ 'Department of Blood Transfusion, \\ The Fourth Hospital of Hebei Medical \\ University, Shijiazhuang, People's \\ Republic of China; ${ }^{2}$ Department of \\ Radiation Oncology, The Fourth \\ Hospital of Hebei Medical University, \\ Shijiazhuang, People's Republic of \\ China; ${ }^{3}$ Animal Center, The Fourth \\ Hospital of Hebei Medical University, \\ Shijiazhuang, People's Republic of \\ China; ${ }^{4}$ Department of Traditional and \\ Western Medical Hepatology, Third \\ Hospital of Hebei Medical University, \\ Shijiazhuang, People's Republic \\ of China
}

Introduction: Hepatocellular carcinoma (HCC) is one of the most common malignant tumors of the digestive system. A disintegrin and metallopeptidase with thrombospondin motif(ADAMTS) has been identified as a secreted metalloproteinase that participates in the inhibition of tumor cell growth and invasion. The aims of the present study were to investigate the clinical significance of ADAMTS8 in patients with HCC and to determine the effect of ADAMTS8 on HCC cell biological activity in vitro and in vivo.

Methods: The tumor tissues and matched adjacent non-tumor tissues were collected from 61 patients with HCC, and ADAMTS8 expression was detected with immunohistochemistry. Flow cytometry and MTT assays were used to assess cell apoptosis and cell viability, respectively, and ERK, p-ERK, Stat3, p-Stat3, Akt, and p-Akt protein expressions were measured by Western blot.

Results: The results showed that ADAMTS8 expression was significantly lower in HCC tissues than that in adjacent non-tumor tissues. Moreover, ADAMTS8 expression was inversely associated with clinical stages and metastasis in patients with HCC. Furthermore, we found that transfection with exogenous ADAMTS8 inhibited proliferation and migration and induced apoptosis in HepG2 cells. In the in vivo study, tumor growth of upregulated HepG2 cells in nude mice was significantly slower. Moreover, decreased ERK activity was detected after transfection with ADAMTS8.

Conclusion: These results indicate that low ADAMTS8 expression is a predictor of a poor prognosis in patients with HCC and that ADAMTS8 plays an important role in regulating HCC growth, invasion, and apoptosis by modulating the ERK signaling pathway. ADAMTS8 maybe a new target in HCC treatment.

Keywords: hepatocellular carcinoma, ADAMT8, apoptosis, invasion and metastasis, signaling pathway

\section{Introduction}

Hepatocellular carcinoma (HCC) is a highly aggressive malignant tumor and represents the fifth most common cause of cancer-related death worldwide. ${ }^{1}$ HCC chemotherapy has clearly improved in past decades; however, the overall survival of patients with HCC remains unsatisfactory. Recurrence, chemotherapy resistance, and metastasis have become the main barriers to improvements in patient survival in the clinical setting. Furthermore, the exact molecular mechanisms underlying HCC progression currently remain unclear. ${ }^{2}$ The identification of the molecules that are involved in HCC progression and affect its prognosis may provide clinicians with effective targets for the clinical treatment of HCC.

A disintegrin and metallopeptidase with thrombospondin motifs (ADAMTSs) are a family of secreted, multi-domain, and extracellular zinc metalloproteinases comprising 19 members in humans. ${ }^{1-3}$ The members of the ADAMTS family have
Correspondence: Yuemin Nan Department of Traditional and Western Medical Hepatology, Third Hospital of Hebei Medical University, I39 Ziqiang Road, Shijiazhuang 0500 I I, People's Republic of China

Tel $+863116678 \quad 227$

Fax +863 II 87023626

Email nanyuemin@।63.com 
one or more thrombospondin type 1 repeat domains in their ancillary regions. ${ }^{2}$ ADAMTS genes participate in a wide range of physiological processes, including extracellular matrix degradation - cell proliferation, apoptosis, migration, and invasion - and angiogenesis ${ }^{3-5}$ in a variety of diseases including thrombotic thrombocytopenic purpura, ${ }^{6,7}$ osteoarthritis, ${ }^{8,9}$ and malignant tumors. ${ }^{4,10,11}$ Recent studies have provided evidence showing that ADAMTS expression is dysregulated in many types of tumors, including gastric, colorectal, pancreatic, lung, esophageal, nasopharyngeal, and breast tumors. ${ }^{12-16}$

ADAMTS8, also known as METH-2, is a novel member of the ADAMTS family and was originally identified as an antiangiogenic factor in a variety of tumors. ${ }^{15,16}$ Genetic and epigenetic analyses have supported the idea that ADAMTS8 acts as an antitumor protease in esophageal squamous cell carcinoma and nasopharyngeal carcinoma. However, the clinical significance and novel roles of ADAMTS8 in HCC remain unclear. Given that ADAMTS8 has inhibitory effects on proliferation and invasion in various tumors, we hypothesize that ADAMTS8 overexpression has similar effects on HCC cells. In this study, the clinical significance and the novel inhibitory effects of ADAMTS8 were evaluated to clarify the roles of the protein in HCC biological activity. Moreover, the underlying mechanisms responsible for the anticancer effects of ADAMTS8 were also investigated. These research findings will provide scientific support for the use of ADAMTS8 as a novel target in clinical HCC treatment.

\section{Methods}

\section{Cell lines and reagents}

Three hepatic carcinoma cell lines (SMMC-7721, HepG2, and Lm-3) and a normal liver cell line (LO-2) obtained from the Animal Center of the Fourth Hospital of Hebei Medical University were purchased from the Institute of Biochemistry and Cell Biology, Chinese Academy of Sciences (Shanghai, China), and the use of the cell lines was approved by the Clinical Research Ethics Committee of the Fourth Hospital of Hebei Medical University. The cell lines were cultured in RPMI-1640 medium (Sigma-Aldrich Co., St Louis, MO, USA) supplemented with 10\% FBS (Thermo Fisher Scientific, Waltham, MA, USA) in a $5 \% \mathrm{CO}_{2}$ humidified incubator at $37^{\circ} \mathrm{C}$. The MTT assay kit was purchased from Sigma-Aldrich Co. The antibodies against ADAMTS8, p-ERK, p-Stat3, p-Akt, and $\beta$-actin were purchased from Abcam (Cambridge, UK). The Annexin V-FITC and 7-AAD double-staining kit was purchased from BD Biosciences
(San Jose, CA, USA). The biotinylated secondary antibody and streptavidin-biotinylated horseradish peroxidase complex were obtained from Zhongshanjinqiao (Beijing, China). Lipofectamine ${ }^{\mathrm{TM}} 2000$ and pPACKH1 $^{\mathrm{TM}}$ Lentivector Packaging Kit were supplied by System Biosciences (Palo Alto, CA, USA).

\section{Liver samples of the patients with HCC}

All biopsy specimens were obtained from patients with liver cancer who were treated in the Fourth Hospital of Hebei Medical University from January 2014 to December 2015. All tumor tissue specimens and corresponding non-tumor tissue specimens from the patients were snap-frozen in liquid nitrogen and stored at $80^{\circ} \mathrm{C}$ for immunohistochemical analysis. The cancer tissues and normal tissues were then routinely stained with $\mathrm{H} \& \mathrm{E}$ stain for pathological observation, and the expression of ADAMTS8 protein in both the cancer and normal tissues was determined by Western blot. All patients and controls gave informed consent to participate in the study, and the patients whose tissues were used in this research provided written informed consent, whose protocol was approved by the Clinical Research Ethics Committee of the Fourth Hospital of Hebei Medical University.

\section{Immunohistochemistry}

ADAMTS8 expression in the HCC tissue specimens was detected by immunohistochemical analysis using the streptavidin-peroxidase method, according to a previous study. ${ }^{17}$ Briefly, the HCC tissue sections were dewaxed, rehydrated, and retrieved, and then they were incubated with primary antibodies against ADAMTS8 for 3 hours at $37^{\circ} \mathrm{C}$ before being incubated with a streptavidin-biotinylated horseradish peroxidase-conjugated secondary antibody. ADAMTS8 protein expression in the HCC tissue sections was visualized and classified based on the staining intensity and the positive cell percentage.

\section{Cell viability assay}

The effect of ADAMTS8 on HCC cell viability was determined by MTT assay. HepG2 cells were plated in 96-well plates (Thermo Fisher Scientific) at a density of $1 \times 10^{4}$ per well. Six duplicate wells were set up for each group. The cells were incubated in $200 \mu \mathrm{L}$ of RPMI-1640 medium containing $10 \% \mathrm{FBS}$. After incubating in a humidified atmosphere for $12,24,36,48,60$, or 72 hours at $37^{\circ} \mathrm{C}$, the cells were treated with $5 \mathrm{mg} / \mathrm{mL}$ MTT solution $(20 \mu \mathrm{L} /$ well $)$ and then incubated for an additional 2 hours at $37^{\circ} \mathrm{C}$. To assess the effect of ADAMTS8 on cell viability, we measured the absorbance 
at $492 \mathrm{~nm}$ using a microplate reader (Titertek Multiskan; Flow Laboratories, North Ryde, Australia). We also show a similar cell viability assay for $3 \mathrm{D}$ cultures to assess the effect of ADAMTS8 using 0.2, 0.4, 0.6, 0.8, 1.0 , and $1.2 \mathrm{mg} / \mathrm{mL}$ antitumor drugs cisplatin (TCI, Shanghai, China).

\section{Flow cytometry assay}

The effect of ADAMTS8 on apoptosis was assessed by flow cytometry analysis. Each group of HepG2 cells were collected after transfection for 48 hours, and washed twice with cold PBS and once in binding buffer, and then incubated with Annexin V-FITC and 7-AAD for 15 minutes, after which they were resuspended in ice-cold PBS and analyzed using a fluorescence-activated cell sorting (FACS) flow cytometer (FACSCalibur ${ }^{\mathrm{TM}}$; Becton Dickinson and Company, Franklin Lakes, NJ, USA). The data were analyzed using CellQuest Pro software.

\section{Tumor cell migration and invasion assays}

Tumor cell migration was assessed using wound healing assay. HepG 2 cells were seeded at a density of $2 \times 10^{4}$ per well. The cell monolayer was scraped with a sterile micropipette tip, and then RPMI-1640 medium supplemented with 2\% FBS was added into six-well culture plates after washing the cells thrice with serum-free medium. The first image of each scratch was acquired at time zero. Each scratch was then reexamined and reimaged at the same location after 48 hours, and the size of the wound area was measured.

The HepG2 cell migration assay was performed in a 24-well transwell chamber (Collaborative Biomedical; Becton Dickinson Labware, Bedford, MA, USA) containing a polycarbonate membrane filter with $8 \mu \mathrm{m}$ pores. The chamber was precoated with $100 \mu \mathrm{g}$ of Matrigel for the invasion assay (Becton Dickinson and Company). Briefly, $2 \times 10^{5}$ HepG 2 cells from different clones were seeded in the upper chamber and incubated in $500 \mu \mathrm{L}$ of RPMI medium (without FBS), while $500 \mu \mathrm{L}$ of medium with $10 \%$ FBS was placed in the lower chamber. The plates were incubated for 24 hours at $37^{\circ} \mathrm{C}$. The cells on the lower surface of the membrane were then fixed in $4 \%$ formaldehyde and stained with $1 \%$ crystal violet in PBS for 5 minutes at room temperature. These cells were considered migrating cells and were counted in five randomly selected fields in each filter at $\times 200$ magnification.

\section{Transfection}

The transfection analyses were performed using a plasmid encoding human ADAMTS8 and a negative control
(Vector). These plasmids labeled with GFP were designed by and purchased from Thermo Fisher Scientific. Cells were stably transfected with the packaged plasmids using Lipofectamine $^{\mathrm{TM}} 2000$ and a pPACKH1 ${ }^{\mathrm{TM}}$ Lentivector Packaging Kit, according to the manufacturer's protocol. Briefly, one day prior to transfection, HepG2 cells (10\%/well) were cultured in six-well tissue culture plates until they reached $50 \%$ confluence. The HepG2 cells were then stably transfected with ADAMTS8 packaged plasmids or empty vectors before being selected with G418 (500 $\mu \mathrm{g} / \mathrm{mL}$; Thermo Fisher Scientific) for 2 weeks. The cells were then collected and used for subsequent experiments.

\section{Western blot analysis}

HepG 2 cells were lysed with $250 \mu \mathrm{L}$ of lysis buffer. The lysates (40 $\mu \mathrm{g}$ per well of the SDS-PAGE gels) were subjected to SDS-PAGE before being electrotransferred onto a polyvinylidene difluoride membrane (EMD Millipore, Billerica, MA, USA), which was incubated with different dilutions of primary antibodies against Akt $(1: 1,000)$, p-Akt $(1: 1,000)$, Stat3 (1:1,000), p-Stat3 (1:1,000), ERK $(1: 1,000)$, p-ERK (1:1,000), p53 (1:1,000), p21 (1:1,000), and $\beta$-actin $(1: 5,000)$ at $4{ }^{\circ} \mathrm{C}$ overnight, followed by incubation with secondary antibody HRP-conjugated anti-mouse IgG (1:5,000 dilution; Thermo Fisher Scientific). Nonspecific antigen binding was blocked by BSA. The membranes were developed using an Odyssey Infrared Imaging System (LI-COR, Lincoln, NE, USA). The expression levels of the target proteins in each sample were normalized to those of $\beta$-actin.

\section{In vivo tumor growth assay}

$\mathrm{Balb} / \mathrm{c}-\mathrm{nu}$ mice were used for the in vivo tumor growth assay. Care was provided according to the National Research Council Guide for the Care and Use of Laboratory Animals, and the experiments were approved by the Institutional Animal Care and Use Committees of Hebei Medical University, China. HepG2 cells from different clones were harvested with trypsin solution and then resuspended in PBS. Cells $\left(1 \times 10^{6}\right.$ cells/mouse $)$ in $0.1 \mathrm{~mL}$ were subcutaneously injected into the null mice $(\mathrm{Balb} / \mathrm{c})$ beginning on the 9th day of the experiment. The mice were randomly divided into the following three groups (10 mice/group): GI, a blank control group treated with parental HepG2 cells; GII, a control-vector group treated with vector-transfected HepG2 cells; and GIII, an ADAMTS8 group treated with ADAMTS8-transfected HepG2 cells. The green fluorescent images for the xenograft were taken using an animal tissue imaging system (Xenogen IVIS Spectrum; PerkinElmer Inc., Waltham, MA, USA) once 
in 2 weeks. The sizes of the tumors were measured, and then all the mice were sacrificed by cervical dislocation during weeks 2 and 4 of the experiment.

\section{Statistical analysis}

Statistical analysis was conducted with the SPSS software package version 13.0 (SPSS, Inc., Chicago, IL, USA). Chisquared tests were used to assess the clinical significance of ADAMTS8 expression. All data are presented as the mean $\pm \mathrm{SD}$ of at least three independent experiments and were analyzed by one-way ANOVA or Student's $t$-test. $P$-values $<0.05$ and 0.01 were considered statistically significant.

\section{Results}

\section{ADAMTS8 expression levels are decreased in HCC tissues}

ADAMTS8 expression in HCC tissues and adjacent nontumor tissues was analyzed by immunohistochemistry. The present study showed that ADAMTS8 was expressed mainly in the cytoplasm and plasmalemma. No staining or only weak staining was observed in the HCC tissue specimens, while high ADAMTS8 expression was observed in the matched adjacent non-tumor tissue specimens. Positive ADAMTS8 expression was noted in 17 (29.8\%) of the 57 HCC specimens and $22(68.8 \%)$ of the adjacent non-tumor tissue specimens. Thus, ADAMTS8 expression was significantly decreased in the HCC tissue specimens compared with the adjacent non-tumor tissue specimens $(P=0.01$, Table 1 , Figures 1 and 4D).

\section{Associations between ADAMTS8 expression and clinicopathological characteristics in patients with HCC}

To characterize the clinical role of ADAMTS8 in HCC, we analyzed the relationships between ADAMTS8 expression in tumor tissues and the clinicopathological parameters of patients with HCC. As shown in Table 2, ADAMTS8 protein expression was not related to gender, age, cirrhosis, tumor size, tumor differentiation, or tumor number but was significantly related to clinical stages and HCC metastasis. Patients with HCC of stage I and II and no metastasis had significantly higher ADAMTS8 expression levels than those with advanced HCC, stage III and IV, and metastasis, indicating that ADAMTS8 expression is inversely correlated with HCC progression. These findings indicate that low ADAMTS8 expression is a predictor of worse clinical disease in patients with HCC.

\section{ADAMTS8 retards proliferation and induces apoptosis in HCC cells}

ADAMTS8 protein expression was assayed in various HCC cell lines, including the LM-3, HepG2, and SMMC-7721 cell lines, and the normal liver cell line LO-5 by Western blotting (Figure 2A and C). ADAMTS8 protein expression was not detectable in HepG2 cells but was detectable in LM-3, SMMC-7721, and LO-5 cells. Thus, these HepG2 cell lines were used in subsequent experiments.

As shown in Figure 2B, ADAMTS8 protein expression was not detectable in blank cells or cells transfected with empty vector. In contrast, HepG2 cells overexpressing ADAMTS8 as a result of transfection showed significantly increased ADAMTS8 protein expression.

To investigate the effect of ADAMTS8 on HCC cell proliferation, we performed MTT assay. As shown in Figure 2D, transfection with ADAMTS8 significantly inhibited HepG2 cell viability in ADAMTS8-transfected cells compared with non-transfected cells and cells transfected with vector alone. Furthermore, a similar cell viability assay using cisplatin further confirmed these results (Figure 4E).

To further assess the inhibitory effects of ADAMTS8 on $\mathrm{HCC}$ cells, we performed flow cytometry to investigate whether overexpressing ADAMTS8 induced HepG2 cell apoptosis. As shown in Figure 2E and F, the percentage of Annexin V-FITC positive cells was significantly increased in the HCC cell lines stably transfected with ADAMTS8 compared to the non-transfected cell lines and the cell lines transfected with vector alone. This finding suggests that the

Table I ADAMTS8 protein expression in HCC tissues and adjacent non-tumor tissues

\begin{tabular}{|c|c|c|c|c|c|}
\hline \multirow[t]{2}{*}{ Group } & \multirow{2}{*}{$\begin{array}{l}\text { Number } \\
\text { of cases }\end{array}$} & \multicolumn{2}{|l|}{ Protein expression } & \multirow{2}{*}{$\begin{array}{l}\text { Positive } \\
\text { expression (\%) }\end{array}$} & \multirow[t]{2}{*}{$P$-value } \\
\hline & & $\begin{array}{l}+ \\
\text { positive }(++\sim++++)\end{array}$ & $\begin{array}{l}- \\
\text { negative }(<++)\end{array}$ & & \\
\hline $\mathrm{HCC}$ tissues & 61 & 19 & 42 & 29.5 & \\
\hline Adjacent tissues & 29 & 20 & 9 & 69.7 & $0.01 *$ \\
\hline
\end{tabular}

Note: $* P<0.05$ was considered to be significant by $\chi^{2}$ test (Fisher's exact test).

Abbreviations: ADAMTS, a disintegrin and metallopeptidase with thrombospondin motif; HCC, hepatocellular carcinoma. 

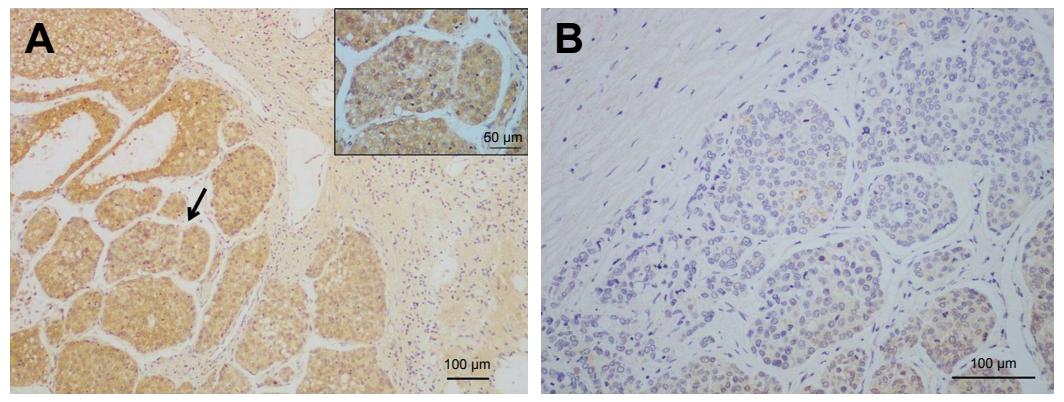

Figure I ADAMTS8 expression in HCC tissues.

Notes: ADAMTS8 protein expression was assessed by immunohistochemical analysis. $\beta$-Actin was used as a loading control. (A) HCC tissues with ADAMTS8 expression $(\times 200)$. Black arrow indicates the particular section for the high-resolution image. $(B)$ HCC tissues without ADAMTS8 expression $(\times 200)$.

Abbreviations: ADAMTS, a disintegrin and metallopeptidase with thrombospondin motif; HCC, hepatocellular carcinoma.

growth suppression observed in these cells was due to an increase in cell death.

\section{ADAMTS8 inhibited HCC cell migration and invasion in vitro}

Migration and invasion are important malignant features of HCC cells. The wound healing experiments revealed that HepG2 cell migration was inhibited after transfection with exogenous ADAMTS8 (Figure 3A and B), and the migration and invasion assay results showed that $98 \pm 6$ cells migrated into the transwell filter after transfection with ADAMTS8. This number of cells was significantly lower than the corresponding numbers of non-transfected and vector-transfected cells that migrated into the filter $(179 \pm 7$ and $191 \pm 4$, respectively) (Figure 3C), suggesting that ADAMTS8 impairs HCC cell migration and invasion ability.

Table 2 Relationship between ADAMTS8 protein expression and the clinicopathological characteristics of patients with HCC

\begin{tabular}{|c|c|c|c|c|c|}
\hline \multirow[t]{2}{*}{ Parameters } & \multirow[t]{2}{*}{ Number } & \multicolumn{2}{|l|}{ ADAMTS-8 } & \multirow[t]{2}{*}{$P$-value } & \multirow[t]{2}{*}{$\chi^{2}$} \\
\hline & & $\begin{array}{l}+ \\
\text { positive }(++\sim++++)\end{array}$ & $\begin{array}{l}- \\
\text { negative }(<++)\end{array}$ & & \\
\hline Gender & & & & 0.501 & 1.46 \\
\hline Male & 49 & 15 & 34 & & \\
\hline Female & 12 & 3 & 9 & & \\
\hline Age (years) & & & & 0.432 & 0.206 \\
\hline$<50$ & 20 & 7 & 13 & & \\
\hline$\geq 50$ & 41 & 12 & 29 & & \\
\hline Clinical stages & & & & $0.026 *$ & 4.78 \\
\hline $\mathrm{I}+\mathrm{II}$ & 42 & 16 & 26 & & \\
\hline III+IV & 19 & 2 & 17 & & \\
\hline Metastasis & & & & $0.030 *$ & 4.51 \\
\hline Yes & 22 & 3 & 18 & & \\
\hline No & 39 & 16 & 23 & & \\
\hline Cirrhosis & & & & 0.455 & 0.138 \\
\hline Yes & 27 & 13 & 14 & & \\
\hline No & 34 & 18 & 16 & & \\
\hline Size, $\mathrm{cm}$ & & & & 0.253 & 0.856 \\
\hline$>5$ & 35 & 19 & 16 & & \\
\hline$<5$ & 26 & 11 & 15 & & \\
\hline Histological grade & & & & 0.399 & 0.337 \\
\hline Good & 12 & 5 & 7 & & \\
\hline Moderate or poor & 49 & 25 & 24 & & \\
\hline Number of tumors & & & & 0.257 & 0.828 \\
\hline Single & 28 & 12 & 16 & & \\
\hline Multiple & 33 & 18 & 15 & & \\
\hline
\end{tabular}

Note: $* P<0.05$ was considered to be significant by $\chi^{2}$ test.

Abbreviations: ADAMTS, a disintegrin and metallopeptidase with thrombospondin motif; HCC, hepatocellular carcinoma. 
A

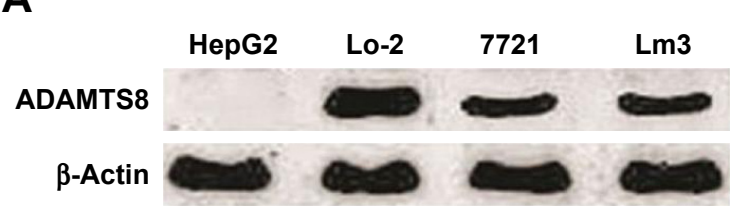

C

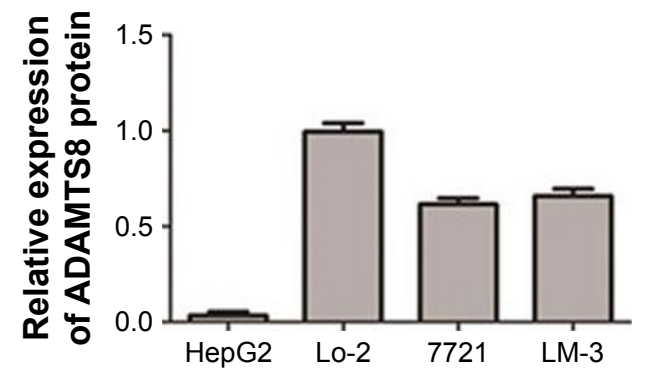

B

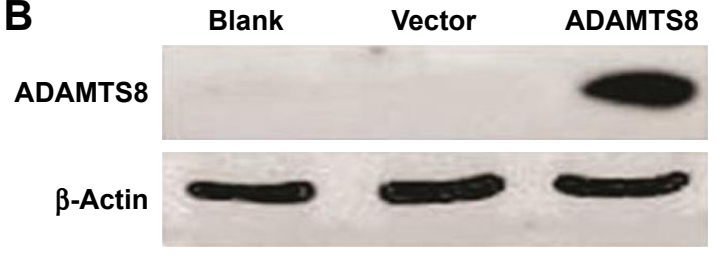

D

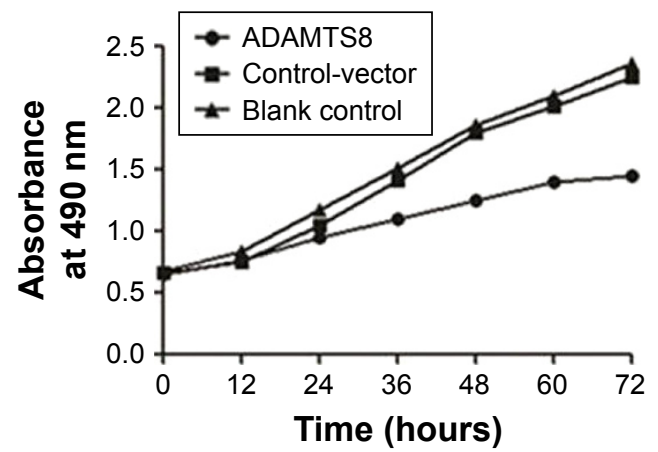

E

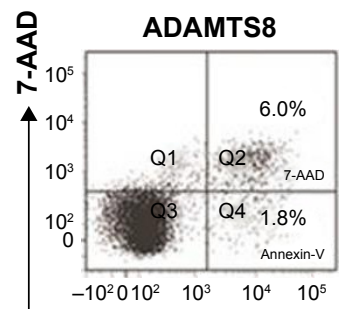

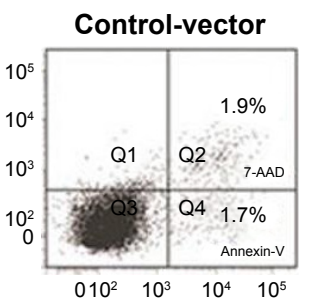

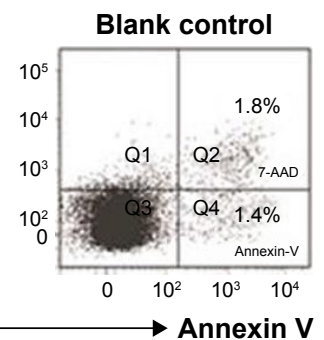

$\mathbf{F}$

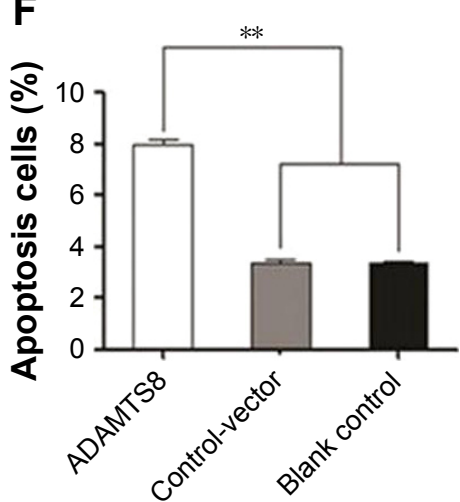

Figure 2 The ability of ADAMTS8 to retard HCC cell proliferation in vitro.

Notes: (A) ADAMTS8 expression levels in different HCC cell lines. (B) ADAMTS8 protein expression in different HepG2 cell clones was analyzed by Western blotting. Blank group: parental cell lines; Vector group: cells stably transfected with vector alone; ADAMTS8 group: cells stably transfected with vector expressing ADAMTS8. (C) Quantification of results of A. (D) The ability of ADAMTS8 to retard HepG2 cell proliferation was analyzed by MTT assay. (E) The ability of ADAMTS8 to induce apoptosis in HepG2 cells was analyzed by flow cytometry. (F) Quantification of results of $\mathbf{E}$. $* * P<0.0$ I compared with the blank control and control-vector groups. Abbreviations: ADAMTS, a disintegrin and metallopeptidase with thrombospondin motif; HCC, hepatocellular carcinoma.

\section{The ERK signaling pathway maybe involved in ADAMTS8-induced HepG2 cell inhibition}

To identify the signaling pathway influenced by ADAMTS8, we evaluated the effect of ADAMTS8 on ERK and Akt/ Stat3 signaling pathway activities. It is well known that both the ERK and Akt/Stat3 signaling pathways play important roles in inhibiting HCC cell growth and inducing HCC cell apoptosis, and tumor suppressor p53 and its major target gene $p 21$ are also involved in cell apoptosis, proliferation. As shown in Figure 4A and B, Western blotting assay showed that $\mathrm{p}$-ERK expression but not $\mathrm{p}$-Akt or $\mathrm{p}$-Stat 3 expression was affected by ADAMTS8 transfection. These data indicate that ADAMTS8 affects the ERK signaling pathway, which regulates HepG2 growth, invasion, and apoptosis (Figure 6); however, the mechanism underlying the effects of ADAMTS8 on the ERK pathway remains unknown.

\section{Effect of ADAMTS8 on HepG2 cell growth in vivo}

In this study, the HCC model was used to evaluate the effect of transfection with endogenous ADAMTS8 on HepG2 cell growth in mice. Growth curve analysis showed that the volumes of tumors overexpressing ADAMTS8 were significantly lower than those of tumors transfected with blanks or control vector $(P<0.01)$ (Figure 5A). The animal tissue imaging system indicated that ADAMTS8-overexpressing tumors were significantly smaller than those transfected with 


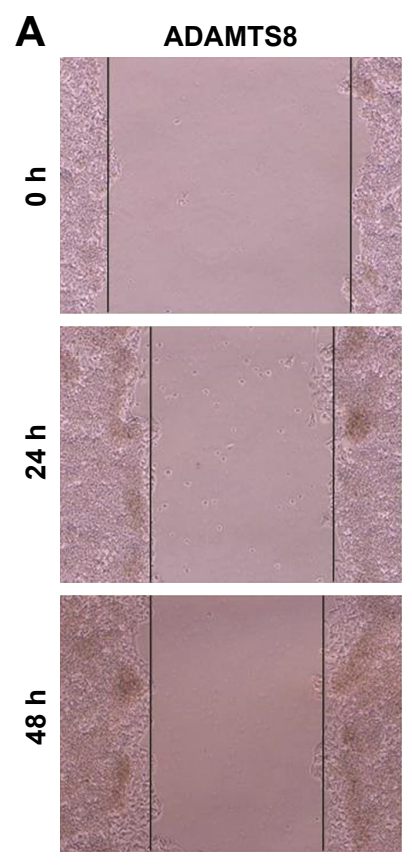

C
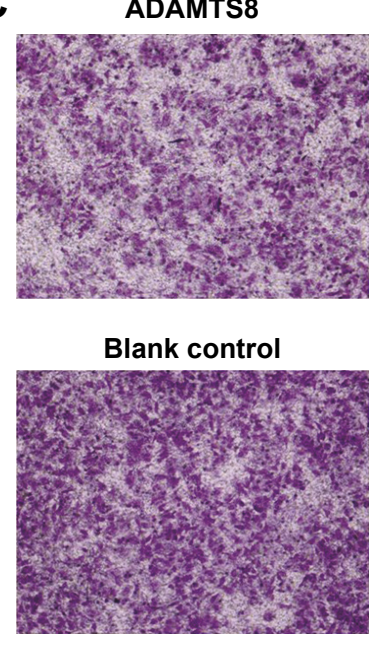

Control-vector
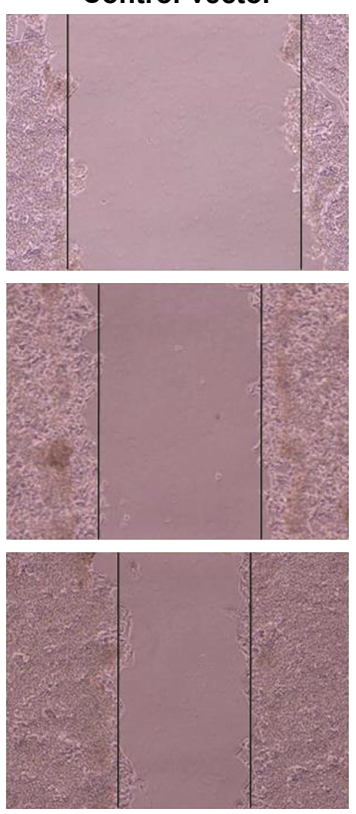

Control-vector
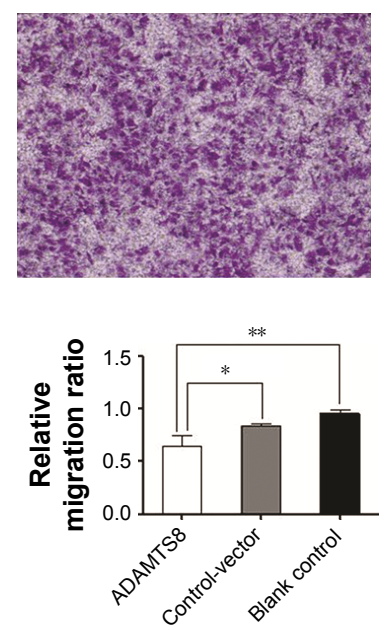

\section{Blank control}
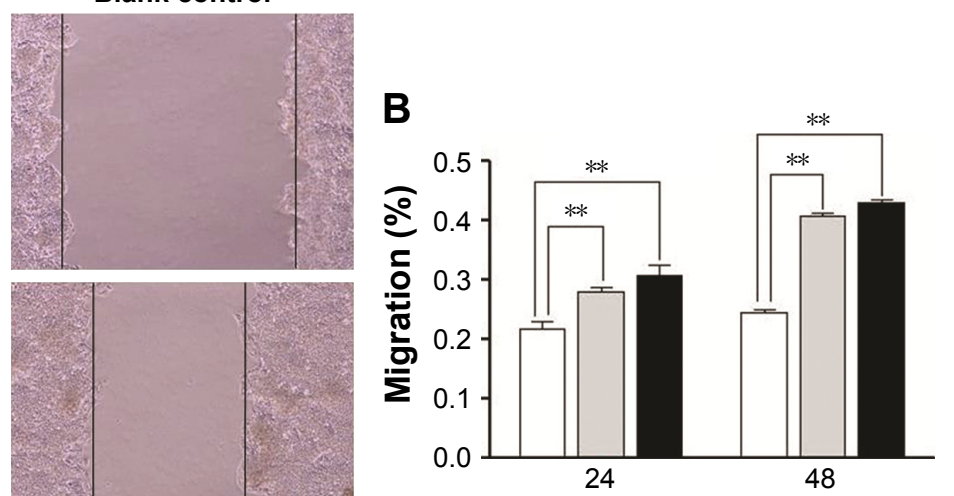

Time (hours)

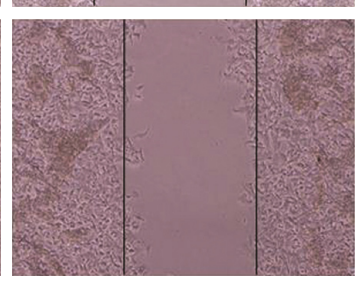

ADAMTS8
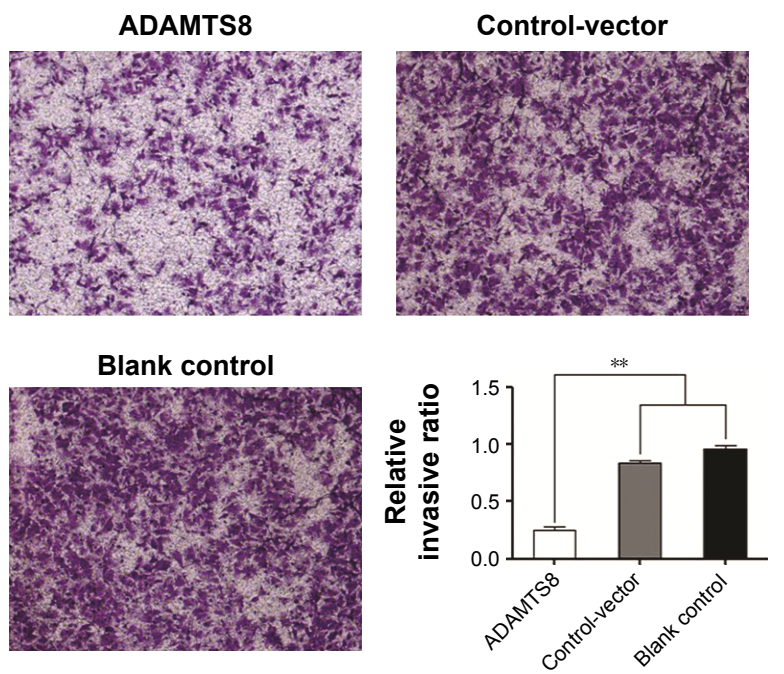

Figure 3 Effects of ADAMTS8 on HCC cell migration and invasion ability.

Notes: (A) The effect of ADAMTS8 on HepG2 cell mobility was investigated by wound healing assay. The wounds were photographed, and the migration percentage in a representative experiment was measured using AxioVision software. $* * P<0.01$ compared with the blank control and control-vector groups. (B) Quantification of results of A. (C) Representative transwell and Matrigel assay images and quantification of migrated and invaded cells. $* P<0.05$, $* * P<0.0$ I compared with the blank control and control-vector groups.

Abbreviations: ADAMTS, a disintegrin and metallopeptidase with thrombospondin motif; HCC, hepatocellular carcinoma.

vector alone (Figure 5B). We analyzed the expression levels of pERK and $\mathrm{p} 53$ in vivo (Figure $4 \mathrm{C}$ ). All of the above results indicate that ADAMTS8 plays an important role in inhibiting HCC cell growth in vivo.

\section{Discussion}

$\mathrm{HCC}$ is one of the deadliest malignant tumors of the digestive system. ${ }^{5}$ Chemotherapy and radiotherapy remain the first-line treatments for HCC. However, HCC metastasis and reoccurrence often lead to treatment failure and patient death. Furthermore, to date, the exact mechanism that induces tumor cell invasion and migration has not been clarified. The development of strategies designed to avoid metastasis remains among the most difficult problems associated with HCC treatment. Investigation of the mechanisms underlying metastasis may provide clinicians with useful targets through which to treat patients with HCC.

ADAMTS8 overexpression reportedly inhibits growth and metastasis by suppressing promoter methylation and angiogenesis in a variety of tumor tissues. Furthermore, 
A

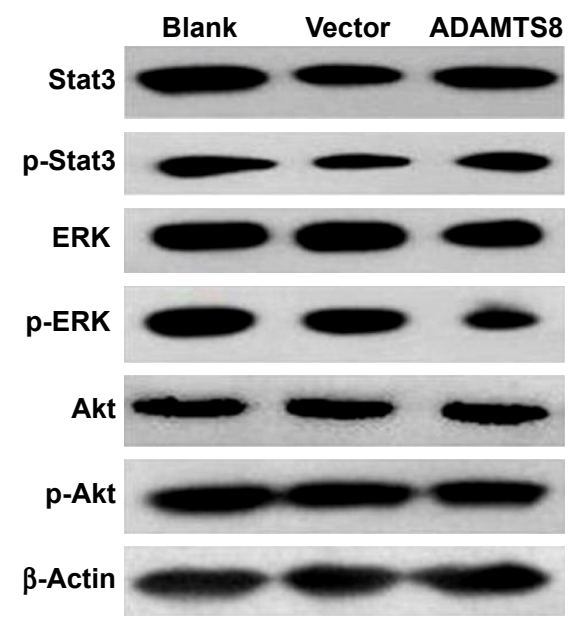

D
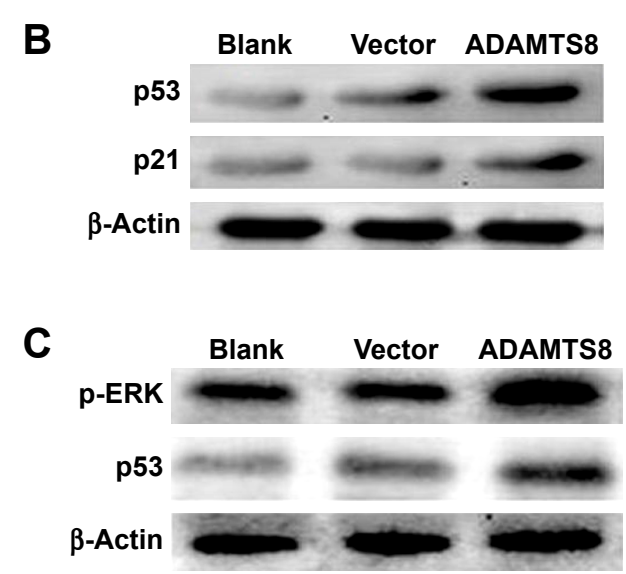

$\mathbf{E}$

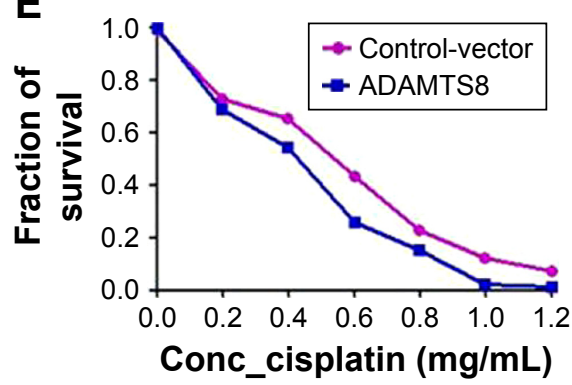

Figure 4 Effects of ADAMTS8 on ERK and Stat3/Akt signaling pathway expressions in HepG2 cells.

Notes: (A) Western blotting assays of ERK, Stat3, Akt, p-ERK, p-Stat3, and p-Akt protein expressions in HepG2 cells. $\beta$-Actin was used as an internal loading control. (B) Western blotting assays of p53 and p2I protein expressions in HepG2 cells. (C) Western blotting assays of p53 and p-ERK protein expressions in vivo. (D) Western blotting assays of ADAMTS8 protein expression in patients with HCC tissues. Line I, 3, 5: HCC tissues and Line 2, 4, 6: corresponding non-tumor tissues. (E) MTT assay analyzed the ability of ADAMTS8 to retard HepG2 cell proliferation using antitumor drug cisplatin.

Abbreviations: ADAMTS, a disintegrin and metallopeptidase with thrombospondin motif; HCC, hepatocellular carcinoma.

it has been reported that higher levels of ADAMTS8 have a poorer clinical prognosis in human breast carcinoma and breast invasive ductal carcinoma. ${ }^{18,19}$ However, the physiological role of the protein is poorly understood..$^{7-9}$ Moreover, to date, the role of ADAMTS8 in HCC metastasis has not been clarified.

In the present study, we analyzed the significance of ADAMTS8 protein expression in HCC tissues and demonstrated that aberrantly low ADAMTS8 expression was negatively correlated with HCC clinical stages and metastasis. Our findings indicated that low ADAMTS8 expression in tumor tissues maybe a predictor of poor prognosis in HCC. These results were in good agreement with those of previous studies on the clinical role of ADAMTS8 in lung and brain malignancies. ${ }^{2,21}$

Furthermore, the MTT assay results showed that transfection with endogenous ADAMTS8 significantly retards HepG2 cell growth, and a similar cell viability assay using cisplatin further verified these results.

Additionally, we observed a significant increase in apoptosis levels in HepG2 cells overexpressing ADAMTS8, suggesting that apoptosis induction maybe involved in ADAMTS8-mediated inhibition of HCC cell proliferation.

Moreover, the wound healing and transwell experiments revealed that HepG2 cell migration and invasion were inhibited after transfection with ADAMTS8, suggesting that the malignant phenotypes of the HCC cell lines are mitigated by ADAMTS8.

To determine the mechanism underlying ADAMTS8mediated apoptosis, we assessed ERK and Akt/Stat3 signaling pathway activities in HepG2 cells transfected with endogenous ADAMTS8. It is well known that ERK and Akt/ Stat3 signaling pathway activities play a key role in regulating the apoptosis of various tumors, including HCC. ${ }^{20,21}$ A previous report showed that ERK signaling pathway activation leads to increases in the expression of the novel anti-apoptosis protein $\mathrm{Bcl}-2$ and the blockage of apoptosis. ${ }^{22,23}$ It is has been reported that increased ADAMTS8 expression suppresses ERK signaling pathway activity by inhibiting EGF protein expression in breast and lung cancers. ${ }^{20-22}$ Overexpressing ADAMTS8 had a similar effect in HCC cells in the present study. Therefore, ADAMTS8 overexpression-induced ERK 

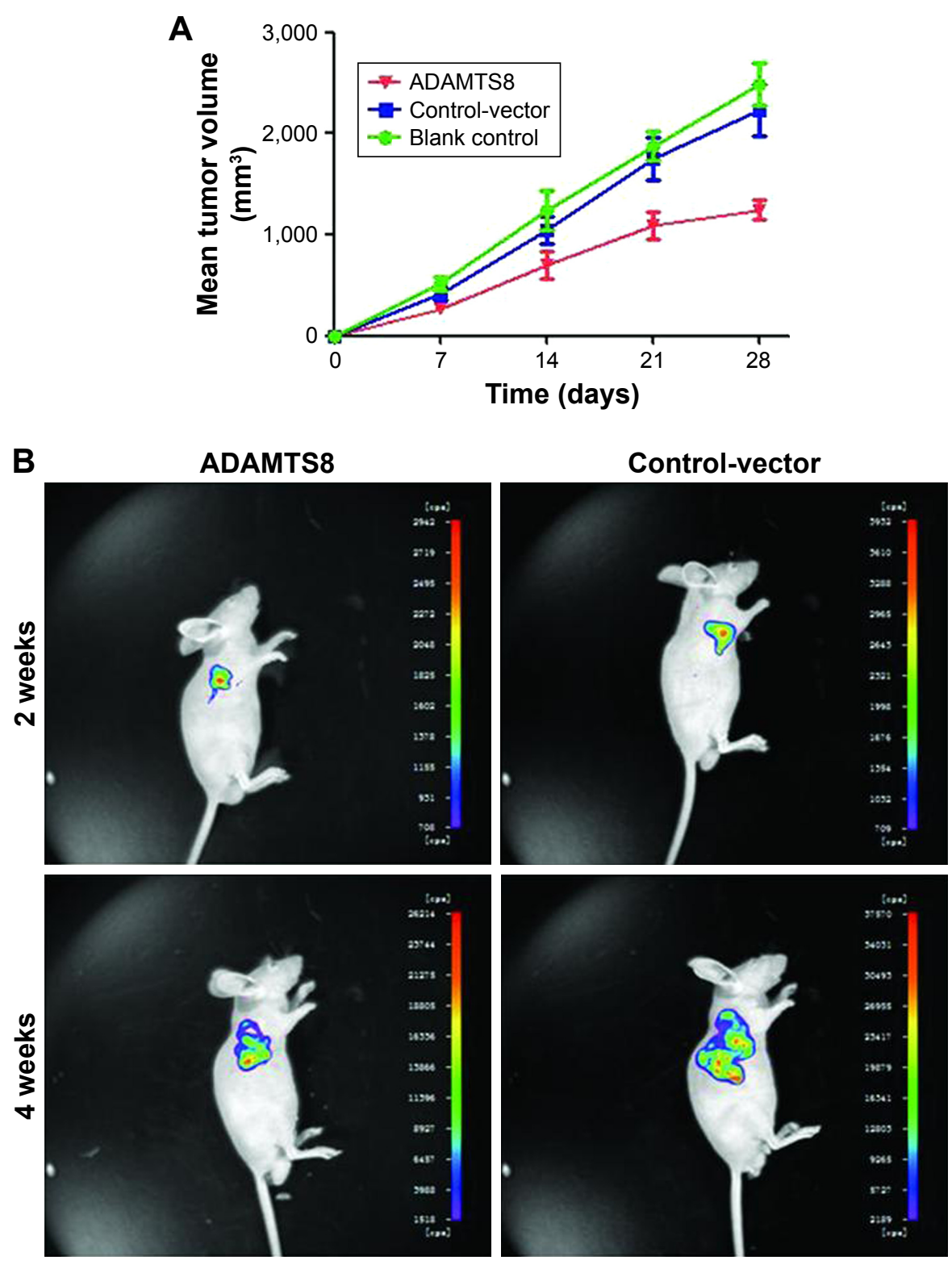

Figure 5 Effects of ADAMTS8 on HCC cell growth in vivo.

Notes: (A) Xenograft tumor growth curves. (B) The tumor tissues were observed using an animal tissue imaging system.

Abbreviations: ADAMTS, a disintegrin and metallopeptidase with thrombospondin motif; HCC, hepatocellular carcinoma.

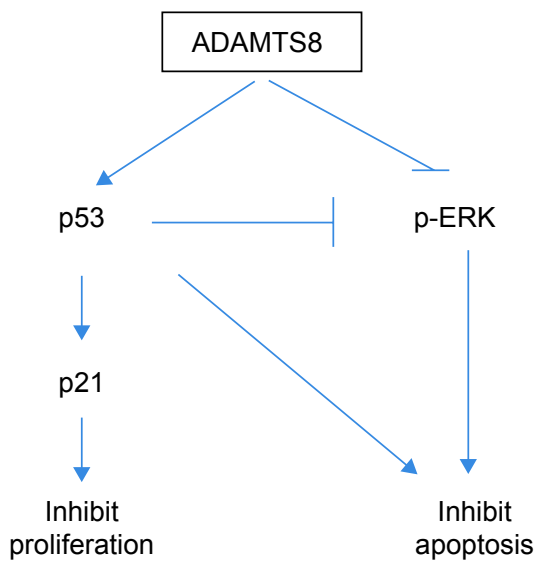

Figure 6 A schematic diagram of the present finding. signaling pathway suppression in HepG2 cells maybe a key factor in improving cell apoptosis levels.

The mouse model showed that in vivo tumor growth was significantly slower in tumors derived from ADAMTS8overexpressing HepG2 cells than in those derived from non-ADAMTS8-overexpressing HepG2 cells, indicating that ADAMTS8-overexpressing xenograft tumors exhibit a relatively benign phenotype.

\section{Conclusion}

In summary, we found that ADAMTS8 inhibits HCC growth and invasion by inducing apoptosis via the ERK signaling pathway. ADAMTS8 modulates ERK activity so as to change 
its transcriptional activity for downstream targets. Therefore, ADAMTS8 maybe a target in the clinical treatment of HCC.

\section{Disclosure}

The authors report no conflicts of interest in this work.

\section{References}

1. Tang BL. ADAMTS: a novel family of extracellular matrix proteases. Int J Biochem Cell Biol. 2001;33(1):33-44.

2. Kumar S, Rao N, Ge R, Rw G. Emerging Roles of ADAMTSs in Angiogenesis and Cancer. Cancers. 2012;4(4):1252-1299.

3. Mochizuki S, Okada Y. ADAMs in cancer cell proliferation and progression. Cancer Sci. 2007;98(5):621-628.

4. Rocks N, Paulissen G, El Hour M, et al. Emerging roles of ADAM and ADAMTS metalloproteinases in cancer. Biochimie. 2008;90(2): 369-379.

5. Tortorella MD, Burn TC, Pratta MA, et al. Purification and cloning of aggrecanase-1: a member of the ADAMTS family of proteins. Science. 1999;284(5420):1664-1666.

6. Camilleri RS, Cohen H, Mackie IJ, et al. Prevalence of the ADAMTS-13 missense mutation R1060W in late onset adult thrombotic thrombocytopenic purpura. J Thromb Haemost. 2008;6(2):331-338.

7. Hughes C, Mcewan JR, Longair I, et al. Cardiac involvement in acute thrombotic thrombocytopenic purpura: association with troponin $\mathrm{T}$ and IgG antibodies to ADAMTS 13. J Thromb Haemost. 2009;7(4): 529-536.

8. Collins-Racie LA, Flannery CR, Zeng W, et al. ADAMTS-8 exhibits aggrecanase activity and is expressed in human articular cartilage. Matrix Biol. 2004;23(4):219-230.

9. Glasson SS, Askew R, Sheppard B, et al. Deletion of active ADAMTS5 prevents cartilage degradation in a murine model of osteoarthritis. Nature. 2005;434(7033):644-648.

10. Turner SL, Blair-Zajdel ME, Bunning RA. ADAMs and ADAMTSs in cancer. Br J Biomed Sci. 2009;66(2):117-128.

11. Porter S, Scott SD, Sassoon EM, et al. Dysregulated expression of adamalysin-thrombospondin genes in human breast carcinoma. Clin Cancer Res. 2004;10(7):2429-2440.
12. Chen J, Zhi Y, Chang X, Zhang S, Dai D. Expression of ADAMTS1 and its correlation with angiogenesis in primary gastric cancer and lymph node metastasis. Dig Dis Sci. 2013;58(2):405-413.

13. Filou S, Korpetinou A, Kyriakopoulou D, et al. ADAMTS expression in colorectal cancer. PLoS One. 2015;10(3):e0121209.

14. Yi JM, Guzzetta AA, Bailey VJ, et al. Novel methylation biomarker panel for the early detection of pancreatic cancer. Clin Cancer Res. 2013;19(23):6544-6555.

15. Dunn JR, Panutsopulos D, Shaw MW, et al. METH-2 silencing and promoter hypermethylation in NSCLC. Br J Cancer. 2004;91(6): $1149-1154$.

16. Jin H, Wang X, Ying J, et al. Epigenetic identification of ADAMTS18 as a novel 16q23.1 tumor suppressor frequently silenced in esophageal, nasopharyngeal and multiple other carcinomas. Oncogene. 2007; 26(53):7490-7498.

17. Ma M, Zhao LM, Yang XX, et al. p-Hydroxylcinnamaldehyde induces the differentiation of oesophageal carcinoma cells via the cAMP-RhoAMAPK signalling pathway. Sci Rep. 2016;6:31315.

18. Porter S, Span PN, Sweep FC, et al. ADAMTS8 and ADAMTS15 expression predicts survival in human breast carcinoma. Int J Cancer. 2006; 118(5):1241-1247.

19. Guo X, Li J, Zhang H, et al. Relationship between ADAMTS8, ADAMTS18, and ADAMTS20 (A disintegrin and metalloproteinase with thrombospondin motifs) expressions and tumor molecular classification, clinical pathological parameters, and prognosis in breast invasive ductal carcinoma. Med Sci Monit. 2018;24:3726-3735.

20. Vázquez F, Hastings G, Ortega MA, et al. METH-1, a human ortholog of ADAMTS-1, and METH-2 are members of a new family of proteins with angio-inhibitory activity. $J$ Biol Chem. 1999;274(33):23349-23357.

21. Dunn JR, Reed JE, du Plessis DG, et al. Expression of ADAMTS-8, a secreted protease with antiangiogenic properties, is downregulated in brain tumours. Br J Cancer. 2006;94(8):1186-1193.

22. Lo PH, Leung AC, Kwok CY, et al. Identification of a tumor suppressive critical region mapping to 3 p14.2 in esophageal squamous cell carcinoma and studies of a candidate tumor suppressor gene, ADAMTS9. Oncogene. 2007;26(1):148-157.

23. Lung HL, Lo PHY, Xie D, et al. Characterization of a novel epigenetically-silenced, growth-suppressive gene, ADAMTS9, and its association with lymph node metastases in nasopharyngeal carcinoma. Int J Cancer. 2008;123(2):401-408.
OncoTargets and Therapy

\section{Publish your work in this journal}

OncoTargets and Therapy is an international, peer-reviewed, open access journal focusing on the pathological basis of all cancers, potential targets for therapy and treatment protocols employed to improve the management of cancer patients. The journal also focuses on the impact of management programs and new therapeutic agents and protocols on

\section{Dovepress}

patient perspectives such as quality of life, adherence and satisfaction. The manuscript management system is completely online and includes a very quick and fair peer-review system, which is all easy to use. Visit http://www.dovepress.com/testimonials.php to read real quotes from published authors. 